\title{
Gamma-rays and neutrinos from accreating neutron stars
}

\author{
Włodek Bednarek \\ Department of Astrophysics, University of Łódź, \\ ul. Pomorska 149/153, 90-236 Eódź, Poland \\ email: bednar@astro.phys.uni.lodz.pl
}

\begin{abstract}
Dense wind of a massive star can be partially captured by a companion neutron star (NS) creating a very turbulent and magnetized transition region at some distance from the NS surface. We consider the consequences of electron and hadron acceleration at such a transition region. Electrons lose energy on the synchrotron process and the inverse Compton (IC) scattering of thermal radiation from the NS surface and/or the massive star. We calculate the synchrotron spectra (from X-rays to soft $\gamma$-rays) and IC spectra in the case of sources accreting the matter under the accretor and propeller scenarios. It is argued that a population of accreting massive binaries, recently discovered by the INTEGRAL observatory, can be detectable by the Fermi LAT telescope. On the other hand, $\mathrm{TeV} \gamma$-ray emission from other class of massive binaries can be interpreted in terms of a magnetar accreting matter in the propeller scenario. We also calculate the expected neutrino event rates in a $\mathrm{km}^{2}$ detector produced by relativistic hadrons accelerated in such scenario.
\end{abstract}

Keywords. Neutron stars, radiation processes, gamma-rays, neutrinos

We consider a compact binary system containing rotating neutron star and a massive companion of the O,B type star. It is assumed that the mass from the companion is effectively captured by a strong gravitational potential of the NS. Depending on the rotational period and surface magnetic field of NS, the accretion process can occur in the phase of accretor, propeller, or ejector. A very turbulent and magnetized transition region is formed when the magnetic pressure balances the pressure of infalling matter. In the case of accretor, most of the matter falls onto the NS surface creating a small hot region on the NS surface (for details see Bednarek 2009a).

The matter penetrate below the light cylinder radius for the NS periods, $P_{\mathrm{I}}>0.084 B_{12}^{4 / 7} M_{16}^{-2 / 7} \mathrm{~s}$, where $B=10^{12} B_{12} \mathrm{G}$ is the surface magnetic field of NS, and $\dot{M}=10^{16} M_{16} \mathrm{~g} \mathrm{~s}^{-1}$ is the accretion rate. The accretion onto NS surface (accretor phase) occurs for the NS periods $P_{\mathrm{II}}>3.7 B_{12}^{6 / 7} M_{16}^{-3 / 7}$ s. The accretion occurs in the propeller phase for $P_{\mathrm{I}}<P<P_{\mathrm{II}}$.

Particles gain energy from the acceleration mechanism, $\dot{P}_{\text {acc }}=\xi c E / r_{\mathrm{L}} \approx 2.6 \times$ $10^{4} \xi_{-1} M_{16}^{6 / 7} B_{12}^{-5 / 7} \mathrm{erg} \mathrm{s}^{-1}$, where $\xi=10^{-1} \xi_{-1}$ is the acceleration parameter, $r_{\mathrm{L}}$ is the Larmor radius of particles, $E$ is the particle energy. Electrons lose energy on the IC process in the Thomson $(\mathrm{T})$ and the Klein-Nishina (KN) regimes. The photon energy density from the polar cap $\left(\rho_{\text {cap }}\right)$ at the acceleration region is, $\rho_{\text {cap }} \approx$ $1.4 \times 10^{8} M_{16}^{11 / 7} B_{12}^{-8 / 7} \mathrm{erg} \mathrm{cm}^{-3}$. The radius of the polar cap region on the NS surface is $R_{\text {cap }}=\left(R_{\mathrm{NS}}^{3} / R_{\mathrm{A}}\right)^{1 / 2} \approx 5 \times 10^{4} B_{12}^{-2 / 7} M_{16}^{1 / 7} \mathrm{~cm}$, and its surface temperature is, $T_{\text {cap }}=$ $\left(L_{\mathrm{X}} / \pi R_{\text {cap }}^{2} \sigma\right)^{1 / 4} \approx 4.7 \times 10^{7} B_{12}^{1 / 7} M_{16}^{5 / 28} \mathrm{~K}$, where $R_{\mathrm{A}}=4 \times 10^{8} B_{12}^{4 / 7} M_{16}^{-2 / 7} \mathrm{~cm}$ is the Alfven radius. The energy density of the magnetic field at $R_{\mathrm{A}}$ is, $\rho_{\mathrm{B}}=B_{\mathrm{A}}^{2} / 8 \pi \approx$ $10^{7} M_{16}^{12 / 7} B_{12}^{-10 / 7} \mathrm{erg}^{-3}$. The energy losses of electrons for the synchrotron and IC 
in the $\mathrm{T}$ regime can be calculated from, $\dot{P}_{\text {loss }}=(4 / 3) c \sigma_{\mathrm{T}} \rho \gamma^{2} \approx 2.7 \times 10^{-14} \rho_{(\text {cap }, \mathrm{B}, \star)} \gamma^{2}$ erg $\mathrm{s}^{-1}$. By balancing energy gains and losses we get the maximum energy of accelerated electrons, $\gamma_{\max } \approx 3 \times 10^{5} \xi_{-1}^{1 / 2} B_{12}^{5 / 14} M_{16}^{-3 / 7}$. The maximum power transfered from the rotating NS to the matter can be estimated on $L_{\text {acc }}=\dot{M}_{\text {acc }} v_{\text {rot }}^{2} / 2 \approx 3 \times 10^{34} B_{12}^{8 / 7} M_{16}^{3 / 7} P_{1}^{-2}$ erg s $\mathrm{s}^{-1}$, where $v_{\text {rot }}$ is the rotational velocity of the magnetic field of NS at $R_{\mathrm{A}}$. The high energy $\gamma$-rays produced in the ICS of thermal photons from the polar cap can be also absorbed in this same radiation field and the massive star radiation initiating an IC $e^{ \pm}$ pair cascade.

We consider $\gamma$-ray production in the accretor and propeller scenarios. Due to the large optical depths, $\gamma$-rays are produced in the IC $e^{ \pm}$pair cascade initiated by relativistic electrons in the radiation of the polar cap. In order to follow the process of $\gamma$-ray production, we developed the numerical code which simulate the cooling process of electrons taking into account not only the $\gamma$-ray production in the IC process but also the synchrotron energy losses of primary electrons and secondary cascade $e^{ \pm}$pairs. Two models for injection of relativistic electrons are considered: (1) injection with the power law spectrum up to the maximum Lorentz factor $\gamma_{\max }$, and (2) monoenergetic injection with the Lorentz factors $\gamma_{\max }$. The example spectra are shown for the sources with different values of $\dot{M}, \xi$ and $B$ and for specific binary IGR J19140+0951 (see Bednarek 2009a). We also consider a neutron star with a superstrong magnetic field accreting the matter in the propeller scenario. The $\gamma$-ray spectrum is calculated from the interaction of electrons with the radiation of the massive star. As an example, we consider the $\mathrm{TeV} \gamma$-ray binary system, LSI +61 303, which was suggested to contain magnetar. The calculations are compared with the X-ray to TeV $\gamma$-ray spectrum of this source (see Bednarek 2009b).

The maximum energies of the accelerated hadrons are determined by the balance between the acceleration time scale and the escape time scale of hadrons from the acceleration site. It can be defined as, $\tau_{\text {esc }}=R_{\mathrm{A}} / v_{\mathrm{f}} \approx 0.4 B_{12}^{6 / 7} M_{16}^{-3 / 7} \mathrm{~s}$, where $v_{\mathrm{f}}$ is the free fall velocity of the matter at $R_{\mathrm{A}}$. We estimate the Lorentz factor of hadrons on, $\gamma_{\mathrm{p}} \approx 2.7 \times 10^{6} \xi_{-1} B_{12}^{1 / 7} M_{16}^{3 / 7}$. We conclude that hadrons are at first accelerated to the maximum energies and after that they interact with thermal photons during their fall onto the NS surface. We calculate the spectra of muon neutrinos coming from the decay of pions produced in collisions of hadrons with the thermal radiation form the NS surface. The expected neutrino event rates from the example sources are reported in Bednarek (2009c).

In summary, we calculated the X-ray to $\gamma$-ray, and neutrino emission from the accreating neutron stars inside the massive binary systems. As an example, we consider the high mass X-ray binary systems: IGR J19140+0951, the TeV $\gamma$-ray binary LSI +61 303, and two X-ray pulsars: GRO 1744-28 and A $0535+262$. We conclude that massive binaries, recently discovered by INTEGRAL, may be detected in the $\gamma$-rays by the Fermi telescope. The accreting magnetar scenario can be responsible for the $\mathrm{TeV} \gamma$-ray emission from LSI +61303 . The nearby X-ray pulsars within binary systems can have a chance to be detected by the future large scale neutrino telescopes.

\section{References}

Bednarek, W. 2009a, A\&\&A 495, 919

Bednarek, W. 2009b, MNRAS 397, 1420

Bednarek, W. 2009c, PRD 79, 123010 